\title{
Microphone Device
}

National Cancer Institute

\section{Source}

National Cancer Institute. Microphone Device. NCI Thesaurus. Code C50068.

A device designed to convert sound to an electrical signal. 